\title{
Gingival Overgrowth on Chronic use of Amlodipine: A Case Report
}

\author{
Muhammed Rashid Parambil Peedikkal', Juny Sebastian ${ }^{1 *}$, Madhan Ramesh ${ }^{1}$, Narayanappa \\ Doddaiah', Sathya Narayana Prashanth ${ }^{2}$
}

1Department of Pharmacy Practice, JSS College of Pharmacy, Jagadguru Shri Shivarathreeshwara University, S S Nagara, Mysuru, INDIA.

${ }^{2}$ Department of Paediatrics, JSS Medical College and Hospital, Jagadguru Shri Shivarathreeshwara University, S S Nagara, Mysuru, INDIA.

\begin{abstract}
Background: Amlodipine, a calcium channel blocker (CCB) which is a commonly prescribed antihypertensive medication. Nifedipine is the CCB which is most frequently associated with drug induced gingival overgrowth. Case report: A 9 year old girl presented to the out-patient department of the hospital with the complaints of swelling of left side of cheek with mild to moderate grade pain since 5-6 days. The pain increased on touching and during jaw movements. Her past medical history revealed that she was a known case of cardiomyopathy with bilateral renal artery stenosis with hypertension and was on oral medications amlodipine ( $5 \mathrm{mg}$ BD), furosemide (40 mg BD) and carvedilol (3.125 mg BD) for the past three years. She was diagnosed as having gingival overgrowth induced by the use of amlodipine. The gingival overgrowth was managed with dental extraction, drainage of abscess and gum cartilage following the withdrawal of the offending drug amlodipine. Alternatively she was started on oral prazosin (1 mg BD) along with other regular medications. Conclusion: Though the incidence of amlodipine causing the gingival overgrowth is less when compared to other drugs in the CCB class, the patients have to be monitored for the gingival overgrowth when on chronic use.
\end{abstract}

Key words: Amlodipine, Calcium channel blocker, Gingival overgrowth, Gum hyperplasia.

\section{INTRODUCTION}

Gingival overgrowth is a kind of painless condition, regarded as enormous enlargement of the inter dental papillae, which can vary from mild to extremely severe pain and can be accompanied by swelling of the gingival margin. ${ }^{1}$ Gingival overgrowth possess a great concern to the physicians and the patients as it is a potential cosmetic problem and a medium for the development of micro organisms. More than 20 drugs are reported to produce the gingival overgrowth as of now. ${ }^{2}$

Three main classes of drugs which majorly induce gingival overgrowth irrespective of their pharmacological effects are anticonvulsants, calcium channel blockers (CCBs) and immunosuppressants. They all exhibit a similar mechanism at the cellular level, where they block the influx of calcium. This blocking effect of above classes of drugs on calcium and sodium ion flux could provide evidence in understanding why three different classes of drugs have a common unpleasant outcome upon a secondary target tissue, such as gingival connective tissue. An evaluation of the range of investigations into the mechanism behind drug-induced gingival overgrowth supports the assumption that it is multifactorial which includes pharmacokinetic, plaque, genetic, biochemical factors, and inflammatory mediators like cytokines, age, sex hormones, and genetic expression of collagens. Plaque scores and gingival inflammation are suggestive to worsen the presentation of drug-induced gingival overgrowth (DIGO). Poor plaque control and degree of plaque induced inflammation are also the contributing factors for the severity of gingival overgrowth irrespective of the drug
DOI: 10.5530/ijopp.10.4.61

Address for correspondence: Juny Sebastian, Department of Pharmacy Practice, JSS College of Pharmacy, Jagadguru Shri Shivarathreeshwara University, S S Nagara, Mysuru-570015, INIDA. Phone no: 9620976279 Email Id: junysebastian@jssuni. edu.in

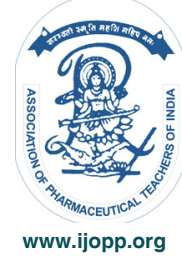


Table 1: Pharmacology and Pharmacokinetics of Amlodipine 5,6

\begin{tabular}{|c|c|}
\hline $\begin{array}{l}\text { Anatomical Therapeutic Chemical (ATC) } \\
\text { Classification code }\end{array}$ & C08CA01 \\
\hline Therapeutic class & Calcium channel blocker \\
\hline Chemical class & Long-acting dihydropyridine \\
\hline Mechanism of action & $\begin{array}{l}\text { Coronary and peripheral arterial vasodilatation by inhibition of } \mathrm{Ca} 2+\text { entry } \\
\text { and blunts the ability of } \mathrm{Ca} 2+\text { to serve as an intracellular messenger. }\end{array}$ \\
\hline Dose & 2.5-5 mg PO qd; Max: 5 mg/day \\
\hline Bioavailability & 60 to $65 \%$ followed by oral administration \\
\hline Peak plasma concentration & 6 to 8hours after administration \\
\hline Protein binding & Up to $98 \%$ \\
\hline Metabolism & In the liver with no significant presystemic or first-pass metabolism \\
\hline Terminal elimination half-life & 40 to 50 hours \\
\hline Adverse effects & Facial blushing, headache, dizziness, gingival overgrowth, oedema \\
\hline
\end{tabular}

intake. $^{3}$

Amlodipine inhibits the trans-membrane influx of calcium ions into vascular smooth muscle and cardiac muscle. The prevalance of amlodipine induced gingival overgrowth is less when compared to other CCBs used in hypertensive and other cardiac disease patients. ${ }^{4}$ The pharmacology and the pharmacokinetic details of amlodipine is present in Table 1

Proper maintenance of oral hygene, dechallenge of the suspected drug and switch over to an alternative, scaling and root planning and the surgical excision of the overgrown gingival tissue are the management options for this ADR. ${ }^{2}$ There are very less reports on gingival hyperplasia followed by chronic use of amlodipine at a dose of $5 \mathrm{mg}$. Here we report our experience of managing gingival hyperplasia in a 9 year old child following the use of amlodipine $5 \mathrm{mg}$ for three years.

\section{CASE REPORT}

A 9 year old female child brought to the hospital with the complaints of swelling of left side of cheek along with mild to moderate grade pain since 5-6 days, dull aching more on touching and during jaw movement. Her past medical history reveals that she is a known case of dilated cardiomyopathy with bilateral renal artery stenosis with hypertension since last three years and was on furosemide(40mg BD), amlodipine(5mg BD), and carvediol(3.125mg BD).

On examination, all her vitals were normal and afebrile. Lower limb pulse was weaker than upper limb and lower limb blood pressure $(110 / 82 \mathrm{mmHg})$ was less than upper limb (125/86 mmHg). Takayasu's arteritis was suspected, which is a chronic inflammatory disorder that involves the aorta, its branches and the pulmonary arteries. The inflammation causes wide degree of stenosis, occlusion or dilatation of the involved vessels. ${ }^{7}$ Loud P2, Parasternal heave, suprasternal and left lower sternal border pulsation were seen. Ejection systolic murmur present (grade III) best heard at mitral area. Abdomen was soft and liver palpable $2 \mathrm{cms}$ below right costal margin.

On examination of the oral cavity, gum hyperplasia with dental caries and left side cheek swelling extending from angle of mandible to 4 X $5 \mathrm{cms}$ medially and downward were observed. Echocardiogram result showed good bilateral ventricular function, mild tricuspid regurgitation and abdominal aortic stenosis. The final diagnosis made was dental abscess with extension into mandible with amlodipine induced gum hypertrophy with dilated cardiomyopathy with Takayasu's arteritis with bilateral artery stenosis and abdominal aorta stenosis with hypertension. The photograph of the gingival overgrowth is present in Figure 1.

As per the advice of Pediatric dentist, $\mathrm{X}$ ray was

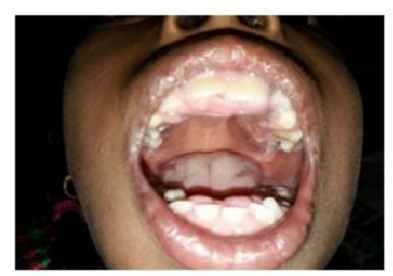

A

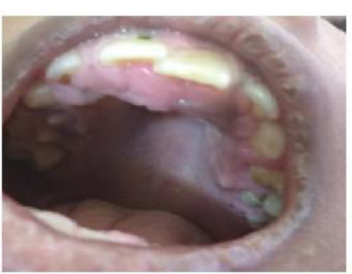

B

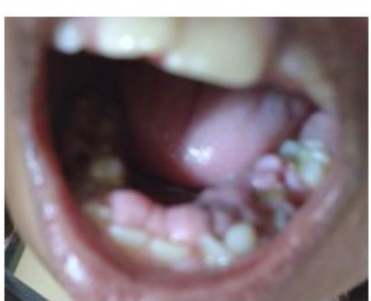

$\mathrm{C}$
Figure 1: Presentation of Gingival overgrowth. 


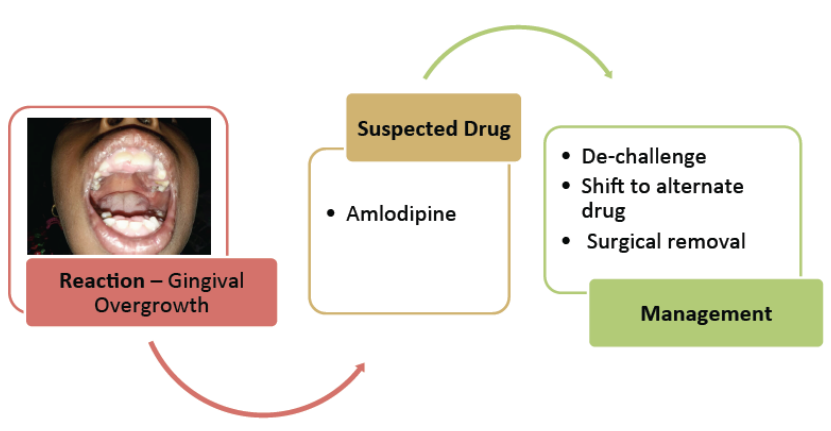

Figure 2: Pictorial presentation of case.

performed which suggested dental abscess which extended to mandible. Paediatric surgeon advised to drain the abscess with dental extraction.

On assessing the case, there was no other risk factor for her gingival overgrowth except the chronic use of amlodipine. The drug was discontinued and put on Injection Linezoild (200mg TID), Injection Amikacin (150mg TID), and Tablet Furosemide 40mg (1/2-1/2-0), Tab.Carvediol3.125mg (1/2-0-1/2) Tab.Methotreaxte $200 \mathrm{mg}$ andfolic acid $5 \mathrm{mg}$ weakly. Case was discussed with paediatric cardiologist and he advised to start Tab. Spironolactone 25mg (1/2-0-1/2). Rheumatologist advised to perform anti-cardiolipin and anti-lupus antibodies and the results were negative. So he advised to hold the use of Tab.Methotrexate and Folic acid.

Second day, Prazocin $1 \mathrm{mg}$ twice daily was started. Dental extraction $(64,65,73,75,84$, and 85$)$ with drainage of abscess and gum cartilage was done after consulting cardiologist and anaesthetist. On third day, she was put on Injection Metronidazole (200mg, TID).Child was dischargedafter ten days as she was stable, taking orally well and swelling and tenderness were subsided. She was put on oral medications paracetamol $300 \mathrm{mg}$, SOS), carvediol (3.125mg, 1/2-0-1/2), furosemide(40mg, $1 / 2-1 / 2-0)$, spironolactone $(25 \mathrm{mg}, 1 / 2-0-1 / 2)$, prazocin (2.5mg, 1/2-0-1/2), lanzoprazole (30mg, $2 / 3$ OD) till next order and metronidazole (200mg, TID) for two more days. Child was advised to review in OPD after five days.The Pictorial presentation of the case is present in Figure 2.

\section{DISCUSSION}

Drug induced gingival overgrowth can be influenced by various factors such as previous medication with its dose, periodontal variables, plaque control, age, genetic factors, sex etc. Among CCBs DIGO is commonly reported with Nifedipine. ${ }^{8}$ Nifedipine has an incidence of $20 \%$, CCBs induced gingival overgrowth prevalence accounts of $38 \%$ and it is 3.3 -fold greater in men when compared to woman. ${ }^{6}$ Amlodipine has prevalence between $1.7-3.3 \%$ in gingival overgrowth. ${ }^{9}$ The mechanisms behind CCBs induced gingival hyperplasia have not been fully understood. Gingival hyperplasia is related by accumulation of steroid intermediate products (androgens) that are transformed to testosterone because of an increase in 17-alpha-hydroxylase enzyme activity. Elevated levels of testosterone may act on the gingival cells and matrix to produce gingival hyperplasia ${ }^{10}$ Irrespective of the drug, severity of gingival enlargement is well associated with plaque control and is proportionate with the level of plaque-induced inflammation. ${ }^{3}$ Drug continuation and the appearance of other risk factors like pharmacokinetic, plaque, genetic and biochemical factors, inflammatory mediators like cytokines, age and sex hormone, and genetic expression of collagens etc, can influence the recurrence of gingival overgrowth.

The calcium antagonist induces blockage of the aldosterone synthesis in zona glomerulosa of the adrenal cortex since this pathway is calcium-dependent, cyclic nucleotide-independent. This may produce a feedback stimulation of an increase in pituitary secretion of ACTH which affects zona glomerulosa hyperplasia.

\section{Assessment of ADR}

The causality assessment was done using both WHO' causality assessment algorithm and Naranjo's scale. Based on a time-temporal relationship and the reaction unlikely attributed to other concurrent disease or medication, it was probable according to the WHO scale and same with Naranjo's scale with a score of 6 . The reaction was not predictable hence not preventable.

\section{CONCLUSION}

It is essential for health care professionals to be aware of the importance of optimal plaque control as it is a major factor for the patients to develop DIGO when on chronic use of Amlodipine. As the ADR is irreversible, switch over to other drugs, maintenance of oral hygeine, and surgical dental extractions are the treatement modalities for the management of DIGO.

\section{ABBREVIATIONS USED}

ADR: Adverse drug reaction; BD: twice daily; DIGO: drug induced gingival overgrowth; CCB: calcium channel blocker; TID: thrice daily; Inj: Injection; Tab: Tablet; CRP: C - reactive protein; ESR: Erythrocyte sedimentation rate; ANA: Anti-nuclear antibody; JVP: 
Jugular venous pressure.

\section{ACKNOWLEDGEMENTS}

The Authors would like to thank the staffs and the post graduate students of department of Pediatctics and department of Clinical Pharmacy, JSS Hospital, Mysuru specially Dr. Harshita Jagwani and Mr. Abhijith Gain for their support and encouragement. .

\section{CONFLICT OF INTEREST}

Nil

\section{REFERENCES}

1. Pavlic V, Zubovic N, llic S, Adamovic T. Untypical Amlodipine-Induced Gingival Hyperplasia. Case Rep, Dent. 2015;756976 [Pubmed].
2. Srivastava AK, Kundu D, Bandyopadhyay P, Pal AK. Management of amlodipine-induced gingival enlargement: Series of three cases. Journal of Indian Society of Periodontology. 2010;14(4):279-81.

3. Vipin B, Chhaya B. Drug-induced gingival overgrowth: The nemesis of gingiva unravelled. Journal of Indian Society of Periodontology. 2013;17(2):182-7.

4. Joshi S, Bansal S. "A rare case report of amlodipine-induced gingival enlargement and review of its pathogenesis". Case Reports in Dentistry. Article ID 138248. 3 pages. 2013.

5. Meredith PA, Elliott HL. Clin-Pharmacokinet. 1992:22(1):22-31.

6. Madi M, Shetty SR, Babu SG, Achalli S. Amlodipine-induced Gingival Hyperplasia-A Case Report and Review. West, I ndian Med J. 2015;64(3):279-82.

7. Kothari S. Takayasu's arteritis in children - a review. Images in Paediatric Cardiology. 2001;3(4):4-23.

8. Carty O, Walsh E, AbdelsalemA, MaCarthy D. Case Report; drug induced gingival overgrowth associated with use of calcium channel blocker (amlodipine). J Ir Dent Assoc. 2015;61(5):248-51.

9. Jorgensen MG. Prevalence of amlodipine-related gingival hyperplasia. J Periodontol. 1997;68(7):676-8.

10. Nyska A, Shemesh M, Tal H, Dayan D. Gingival hyperplasia induced by calcium channel blockers: mode of action. Med Hypotheses. 1994;43(2):115-8. 